\title{
Outcomes of community-based HIV testing modalities in a Mpumalanga district, South Africa
}

\author{
A Bosman, ${ }^{1}$ BCom (Fin Man), HED, BCom Hons (Acc), ACHM, MPH; \\ A Beke, ${ }^{2} \mathrm{MB}$ ChB, MMed (Comm Health), FCPHM (SA), FGCP, DTM\&H, DPH, DHSM, DOH \\ ${ }^{1}$ School of Health Systems and Public Health, Faculty of Health Sciences, University of Pretoria; and Technical Assistance Cluster, Foundation for \\ Professional Development (Pty) Ltd, Pretoria, South Africa \\ ${ }^{2}$ School of Health Systems and Public Health, Faculty of Health Sciences, University of Pretoria, South Africa
}

Corresponding author: A Bosman (aletb@foundation.co.za)

\begin{abstract}
Background. Testing for HIV in individuals informs them of their HIV status, which in turn enables them to receive prevention, treatment and care services. HIV services should ideally be accessed before an individual's immune system is severely damaged, which could also increase the prevention of new HIV infections. The national HIV testing services (HTS) programmes aim to identify HIV-infected individuals and link them to prevention, care and treatment services. Community-based (CB) HTS reaches community members who do not have access to such services at healthcare facilities. The Foundation for Professional Development (FPD) provided CB-HTS in a Mpumalanga district, South Africa, from 1 October 2016 to 30 September 2017 (Country Operating Plan (COP16)), where 65691 clients were tested.

Objectives. To determine which of the FPD CB-HTS modalities used in the Mpumalanga district during COP16 delivered the highest positivity rate, disaggregated by population segmentation. The accompanying objectives were: to describe the demographic characteristics of HTS clients in the district and to compare the different positivity rates of FPD CB-HTS modalities in the district.

Methods. This cross-sectional quantitative study used all the individual, programmatic data collected for all CB-HTS clients in Ehlanzeni during COP16 as secondary data. Descriptive analysis was employed to describe participants' characteristics. The $\chi^{2}$ test was used for comparing variables.

Results. The mean age of clients was 29.3 (95\% confidence interval (CI) 29.7 - 29.9) years. Of the clients analysed, $56.4 \%$ were females. Of the clients who were tested for HIV, $14.1 \%$ were tested for the first time; $67.7 \%$ of those were between 15 and 49 years old. The positivity rate for each modality was calculated, and it was found that home-based HTS had the best positivity rate (9.1\%) in the Mpumalanga district during COP16.

Conclusions. This study provides evidence that home-based HTS delivered the best positivity rate in the Mpumalanga district. The results should be used to replicate the programme in other districts. If similar findings are obtained in other studies, it could inform how future CB-HTS programmes should be rolled out, which could change future strategic planning and resource allocation for CB-HTS programmes.
\end{abstract}

S Afr Med J 2020;110(10):1041-1044. https://doi.org/10.7196/SAMJ.2020.v110i10.14417

In 2011, 34.2 million individuals worldwide were living with HIV. ${ }^{[1]}$ The number had increased to 36.7 million in 2016. ${ }^{[2]}$ In 2012, 23.5 million HIV-infected individuals were from Africa. ${ }^{[1]}$ Only $12 \%$ of the total worldwide population was living in sub-Saharan Africa in 2013, but the region accounted for $71 \%$ of the global HIV burden ${ }^{[3]}$ Despite the burden of the disease, only a third of adults in sub-Saharan Africa were tested for HIV in 2012. ${ }^{[4]}$ The total number of HIV-infected individuals in South Africa (SA) was estimated to be 6.4 million in $2012 .{ }^{[1]}$ It was also estimated that $23.7 \%$ of South Africans were undiagnosed in 2012. ${ }^{[5]}$ It is not possible for an individual to receive prevention, treatment and care services if they do not know their HIV status, i.e. whether it is positive or negative. ${ }^{[6]}$ Furthermore, an early diagnosis enables individuals to access healthcare before their immune systems are severely damaged, and enables the prevention of new HIV infections. ${ }^{[7]}$ Individuals also tend to reduce their high-risk behaviour once they know their HIV status. ${ }^{[8]}$

The aim of a national HIV testing services (HTS) programme is, according to the consolidated guidelines on the use of antiretroviral drugs for treating and preventing HIV infection, to 'identify as many people as possible with HIV early in their infection and link them successfully to prevention, care and treatment services. ${ }^{[9]}$ HTS provided at healthcare facilities is complemented by communitybased (CB) HTS modalities and is implemented in high-incidence communities close to where people live and work. CB-HTS also aims to reach communities that do not have access to HTS at health facilities and to reach HIV-infected individuals earlier on in the course of the disease. ${ }^{[10]}$

The SA government has facilitated the testing for HIV, and thus increased the proportion of people who have been tested and know their HIV status - from $50 \%$ in 2008 to $66.5 \%$ in $2014 .{ }^{[11]}$ The implementation of the World Health Organization (WHO) guidelines of universal test and treat (UTT) on 1 September 2016, was another milestone in the SA government's effort to scale up HTS. According to the UTT guidelines, an individual should be initiated on antiretroviral treatment (ART) as soon as possible and within 2 weeks of their CD4+ count being done. The Foundation for Professional Development (FPD) was awarded a grant by the US Agency for International Development (USAID) to implement a CB-HTS programme in 2014. The FPD CB-HTS programme used home-based HTS, index client trailing and mobile HTS. Homebased HTS provides an opportunity for adults, families and couples to be tested in their homes by lay counsellors. ${ }^{[12]}$ Mobile HTS makes it possible to access hard-to-reach and high-risk populations. ${ }^{[13]}$ 
Mobile HTS units can perform HIV tests in both urban and rural populations. When these units perform HIV tests at a workplace, it is classified as 'workplace' HTS. Index client trailing focuses on HIV-infected individuals and offers to test their household and family for HIV, including their children. ${ }^{[14]}$

During the USAID Country Operating Plan of 1 October 2016 30 September 2017 (COP16), the FPD CB-HTS programme (FPD CB-HTS) tested 603364 individuals. During COP16, the programme was active in 27 high-burden sub-districts or local municipalities in 13 districts, which are situated in 6 provinces. The programme made use of home-based HTS, index client trailing, mobile HTS and workplace modalities. The workplace modality did not include schools, but did include workplace implementation modalities. A total of 65691 individuals were tested in a district in Mpumalanga during COP16. The number of individuals tested differed between districts and provinces, e.g. a district in Mpumalanga tested 65691 individuals, while a district in KwaZuluNatal tested 23993 individuals. These differences between districts highlight the importance of identifying the most relevant testing modality for different communities to maximise HIV testing and counselling coverage and access. ${ }^{[15]}$ Such knowledge will inform HTS providers regarding how to make optimal use of restricted HIV testing facilities in the face of stock-outs of test kits and a shortage of healthcare workers. The aim of this study, based on the shortage of resources, was to determine which of the FPD CB-HTS modalities used in the Mpumalanga district during COP16 delivered the highest positivity rate, disaggregated by population segmentation. Positivity rate is defined as the number of confirmed HIV cases per 100 suspected HIV cases examined, and provides an alternative method to estimate changes in incidence. ${ }^{[16]}$ The district in Mpumalanga was selected for this study, as the captured data have been audited for correctness.

\section{Methods}

The study employed a cross-sectional quantitative design. Individual, programmatic data collected for FPD CB-HTS clients in the Mpumalanga district during the COP16 were used as secondary data. Data for each of the FPD CB-HTS clients were collected according to the FPD CB-HTS data collection plan. SA Government HTS registers and consent forms were used as main data collection sources. FPD CB-HTS programme managers were responsible for supervising data collection, in line with requirements of the data collection plan. All data collected and captured were evaluated for accuracy, validity, reliability, timeliness, precision and integrity to ensure data quality. All relevant staff received standardised training on data quality. The review of captured data included spot checks, count and verification exercises, reviewing data logics for errors and anomalies, reviewing data against understood programme performance expectations and conducting structured data quality audits.

The results of 65691 FPD CB-HTS clients in the Mpumalanga district were captured on the database for COP16. During data cleaning, 434 results were deleted, as they were incomplete owing to the test result not being available $(n=89)$, a conclusion that could not be made regarding the clients' HIV status $(n=202)$, the date of birth that was incorrectly captured $(n=121)$ and the modality used not being captured correctly $(n=22)$. The remaining 65257 FPD CB-HTS client results were used for analysis.

Descriptive statistics, including mean, standard deviation, median, interquartile range and frequency distribution, were used to describe participants' characteristics. The $\chi^{2}$ statistical test was used for categorical variables. Proportions related to uptake, the corresponding 95\% confidence interval (CI) and probability of 0.5 were calculated. The HIV positivity rate was calculated as the number of HIV-infected clients per total number of clients tested and reported as a percentage. All analyses were conducted with Stata 14.0 (Stata Corp., USA).

\section{Ethical approval}

FPD, the owner of the data, granted approval and provided the data for the study. The study was approved by the Faculty of Health Sciences Research Ethics Committee, University of Pretoria (ref. no. 401/2018).

\section{Results}

The results of 65257 FPD CB-HTS clients during COP16 were analysed. Of these clients, 36810 (56.4\%) were females and 28447 (43.6\%) were males. Clients' birth dates were used to calculate their age on the date that they were tested and approximated a normal distribution. The mean age of clients was 29.8 (95\% CI 29.7 - 29.9) years. The ages of clients on the date that they were tested were converted into categorial variables and grouped for every 5 years. The age and gender distribution of clients was compared with the testing modalities used by clients (Table 1). Index client trailing (57.4\%), home-based HTS (56.6\%) and mobile HTS (56.4\%) were more popular with female clients. Workplace HTS was preferred by males (51.9\%).

The total number of CB-HTS clients analysed ( $n=65256)$ included $14.1 \%$ ( $n=9217)$ who were tested for HIV for the first time (Table 2). One of the clients who was between 15 and 19 years old when tested was omitted from the calculation, because it was not specified if the client was a first-time tester. The total number of clients used for this analysis was therefore 65256 . Of the clients tested for the first time, $67.7 \%$ were between 15 and 49 years old.

The majority of clients $(78.7 \%)$ who received an HIV test for the first time used the home-based HTS (Table 3), followed by mobile HTS, which was the second most used service (18.2\%).

There was no statistically significant difference between the modalities and HIV test results $\left(\chi^{2}=3.2 ; p=0.361\right)$. The positivity rate for each modality was calculated by dividing the number of HIVinfected clients per modality by the total number of clients tested (Table 4). Home-based HTS had the best positivity rate (9.1\%) for FPD CB-HTS clients in the Mpumalanga district during COP16. Descriptive analyses conducted on the raw FPD CB-HTS data for the district, initially indicated that index client trailing was the testing modality that delivered the best positivity rate.

\section{Discussion}

Very few articles comparing HTS modalities and the positivity rate of CB-HTS modalities have been published to date, especially articles on CB-HTS programmes in SA. The 4 HTS delivery models reviewed and discussed by Mabuto et al. ${ }^{[17]}$ were clinic-based, stand-alone and urban mobile and rural mobile HTS. The study by Mabuto et al. ${ }^{[17]}$ did not include home-based and index client trailing, but it did calculate the positivity rate of its 4 modalities. Naik et al. ${ }^{[18]}$ who conducted a home-based HTS in KwaZulu-Natal in 2011 , found that $\sim 9.7 \%$ of clients tested were HIV-infected. ${ }^{[18]}$ The positivity rate from the study by Naik et al. ${ }^{[18]}$ is in line with that for home-based HTS in this study. Another study in KwaZuluNatal in 2016 found that home-based HTS could contribute to a lower HIV incidence, especially if it results in linkage to care. ${ }^{[19]}$ Home-based HTS delivered the highest positivity rate in this study 


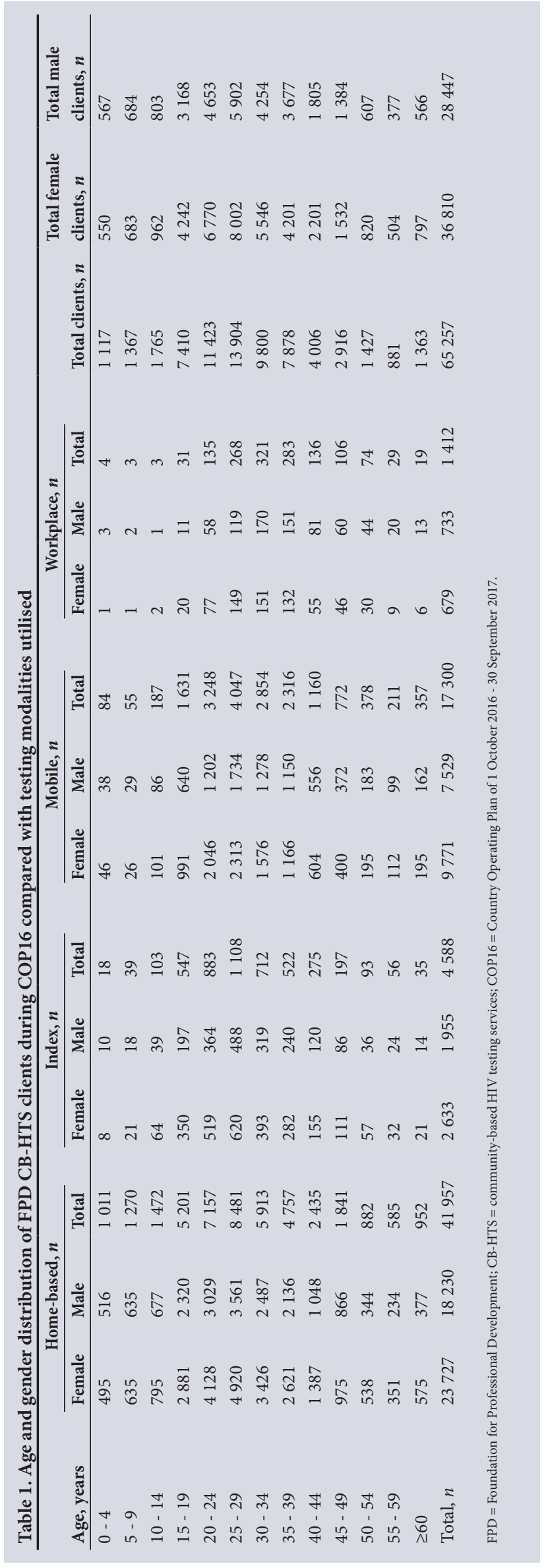

Table 2. Distribution of first-time testers

\begin{tabular}{llll}
\hline Age, years & $\begin{array}{l}\text { Previously } \\
\text { tested, } \boldsymbol{n}\end{array}$ & $\begin{array}{l}\text { First time } \\
\text { tested, } \boldsymbol{n}\end{array}$ & Total, $\boldsymbol{n}$ \\
\hline $0-4$ & 452 & 665 & 1117 \\
$5-9$ & 474 & 893 & 1367 \\
$10-14$ & 797 & 968 & 1765 \\
$15-19$ & 5558 & 1851 & 7409 \\
$20-24$ & 10148 & 1275 & 11423 \\
$25-29$ & 12618 & 1286 & 13904 \\
$30-34$ & 9082 & 718 & 9800 \\
$35-39$ & 7268 & 610 & 7878 \\
$40-44$ & 3730 & 276 & 4006 \\
$45-49$ & 2693 & 223 & 2916 \\
$50-54$ & 1289 & 138 & 1427 \\
$55-59$ & 795 & 86 & 881 \\
$\geq 60$ & 1135 & 228 & 1363 \\
Total, $n$ & 56039 & 9217 & 65256 \\
& & &
\end{tabular}

and could therefore contribute to a lower HIV incidence. A study in Swaziland found that the positivity rate among home-based HTS clients was $3.5 \%$, whereas it was $4.7 \%$ among mobile HTS clients. ${ }^{[20]}$ This study had a positivity rate of $9.1 \%$ among home-based HTS clients and 8.8\% among mobile HTS clients, which are both higher than the results from the Swaziland study.

It is estimated that adult men, aged $\geq 15$ years, comprise $37 \%$ of all HIV-infected adults in SA. ${ }^{[21]}$ The current study found that $43.6 \%$ of CB-HTS clients were males, of whom $92.8 \%$ were $\geq 15$ years. This may indicate that CB-HTS is a more effective method to test adult men than facility-based HTS. The majority $(87.9 \%)$ of CB-HTS clients were 15 - 49 years old - the age group where most new HIV infections are diagnosed. ${ }^{[22]}$ CB-HTS therefore reaches the age group most at risk. The majority $(67.7 \%)$ of clients tested for the first time also fell in this age group.

The positivity rate between the different FPD CB-HTS modalities does not differ $>1 \%$ between the highest modality, home-based HTS with a $9.1 \%$ positivity rate, and the lowest modality, index client trailing with an $8.3 \%$ positivity rate. The average FPD CB-HTS positivity rate of $8.9 \%$ was consistent with that in the study by Mabuto et al., ${ }^{[17]}$ which obtained a $9.3 \%$ positivity rate. The study found that home-based HTS was the most effective FPD CB-HTS modality in terms of positivity rate in the district being studied. The FPD CB-HTS home-based testing modality also proved to be the preferred testing modality among first-time testers.

\section{Conclusions}

This study has proven that the FPD CB-HTS modalities performed well in the district in Mpumalanga, with home-based HTS delivering the highest positivity rate. The vulnerable age group of $15-49$ years was mainly reached by FPD CB-HTS, which could contribute to the lower HIV incidence. If this study is replicated in other districts, it could inform how future resource allocation should be rolled out and planned. It could also potentially change future strategic planning and resource allocation for CB-HTS programmes.

A similar study could also be used to compare provinces or districts per HIV burden.

\section{Study limitations}

The data used in this study were not collected by the researcher or for research purposes, but for programme implementation and 


\begin{tabular}{llll} 
Table 3. Modalities preferred by first-time testers & & \\
\hline Testing modality & Previously tested, $\boldsymbol{n}$ & First time tested, $\boldsymbol{n}$ & Total, $\boldsymbol{n}$ \\
\hline Home-based & 34707 & 7250 & 41957 \\
Mobile & 15624 & 1675 & 17300 \\
Index client trailing & 4390 & 198 & 4588 \\
Workplace & 1318 & 94 & 1412 \\
Total & 56039 & 9217 & 65257
\end{tabular}

Table 4. Positivity rate of the FPD CB-HTS modalities

\begin{tabular}{|c|c|c|c|c|c|}
\hline \multirow[b]{2}{*}{ HIV status } & \multicolumn{4}{|c|}{ Testing modality } & \multirow[b]{2}{*}{ Total, $n$} \\
\hline & Home based & Mobile & Index & Workplace & \\
\hline HIV-positive & 3800 & 1525 & 382 & 124 & 5831 \\
\hline HIV-negative & 38157 & 15775 & 4206 & 1288 & 59426 \\
\hline Total & 41957 & 17300 & 4588 & 1412 & 65257 \\
\hline Positivity rate, \% & 9.06 & 8.82 & 8.33 & 8.78 & 8.94 \\
\hline
\end{tabular}

reporting. The researchers therefore had to rely on programme managers for accuracy of the data, which could limit the accuracy of the study findings. The study was conducted in one district in SA and may not be representative of other districts in the country.

\section{Declaration. None.}

Acknowledgements. We thank Dr Nkhensani Nkhwashu and Mr Jean Slabbert of the Foundation for Professional Development (FPD), who provided the data used in this study.

Author contributions. A Bosman: conceptualised the study, conducted the analysis, wrote the first draft of the article, and provided guidance on the analysis. Both authors contributed to the study design, data interpretations and final revisions to the text.

Funding. The FPD community-based HIV testing services programme was funded by the President's Emergency Plan for AIDS Relief (agreement number AID-674-A-14-0006).

Conflicts of interest. None.

1. Shisana O, Rehle T, Simbayi LC, et al. South African National HIV Prevalence, Incidence and Behaviour Survey, 2012. Cape Town: HSRC Press, 2014.

2. World Health Organization. Global health observatory (GHO) data. 2017. http://www.who.int/gho/ hiv/en/ (accessed 25 August 2020).

3. Kharsany AB, Karim QA. HIV infection and AIDS in sub-Saharan Africa: Current status, challenges and opportunities. Open AIDS J 2016;10:34-48.

4. Joint United Nations Programme on HIV and AIDS. Fact Sheet. Global Statistics. Geneva: UNAIDS, 2014 5. Johnson LF, Rehle TM, Jooste S, Bekker LG. Rates of HIV testing and diagnosis in South Africa: Successes and challenges. AIDS 2015;29(11):1401-1409. https://doi.org/10.1097/QAD.0000000000000721

6. World Health Organization. Service Delivery Approaches to HIV Testing and Conselling (HTC): A Strategic HTC Programme Framework. Geneva: WHO, 2012.

7. Tooley L. Detecting HIV earlier. Adv HIV Test 2016;23(11):24-25.

8. US Department of Veterans Affairs. HIV/AIDS. https://www.hiv.va.gov/provider/topics/testing-earlydiagnosis.asp (accessed 28 May 2018).
9. World Health Organization. Consolidated Guidelines on the Use of Antiretroviral Drugs for Treating and Preventing HIV Infection: Recommendations for a Public Health Approach. Geneva: WHO, 2016.

10. Foundation for Professional Development. Community-based HIV testing services. http://www. foundation.co.za/hiv-testing (accessed 12 February 2018).

11. Southern African HIV Clinicians Society. Implementation of the universal test and treat strategy for HIV positive patients and differentiated care for stable patients. http://www.sahivsoc.org/ SubHeader?slug=ndoh-and-who-guidelines (accessed 19 March 2018).

12. Van Rooyen H, Barnabas RV, Baeten JM, et al. High HIV testing uptake and linkage to care in a novel program of home-based HIV counseling and testing with facilitated referral in KwaZuluNatal, South Africa. J Acquir Immune Defic Syndr 2013;64(1):e1-e8. https://doi.org/10.1097/ QAI.0b013e31829b567d

13. Govindasamy D, van Schaik N, Kranzer K, Wood R, Mathews C, Bekker LG. Linkage to HIV care from a mobile testing unit in South Africa by different CD4 count strata. J Acquir Immune Defic Syndr 2011:58(3):344-352. https://doi.org/10.1097/QAI.0b013e31822e0c4c

14. National Department of Health. National HIV Testing Services: Policy and Guidelines, 2015. Pretoria: NDoH, 2015.

15. HIV Testing and Counseling (HTC). Global Forum on MSM and HIV. http://msmgf.org/ files/msmgf/documents/TechBulletins/EN/Sec7MSMGF_TechBulletins2012.1.pdf (accessed 28 May 2018)

16. Jensen TP, Bukirwa H, Njama-Meya D, et al. Use of the slide positivity rate to estimate changes in malaria incidence in a cohort of Ugandan children. Malar J 2009;8(1):213. https://doi. org/10.1186/1475-2875-8-213

17. Mabuto T, Latka MH, Kuwane B, Churchyard GJ, Charalambous S, Hoffmann CJ. Four models of HIV counseling and testing: Utilization and test results in South Africa. PLoS ONE 2014;9(7):e102267. https://doi.org/10.1371/journal.pone.0102267

18. Naik R, Doherty T, Jackson D, et al. Linkage to care following a home-based HIV counselling and testing intervention in rural South Africa. J Int AIDS Soc 2015;18(1). https://doi.org/10.7448/ IAS.18.1.19843

19. Roberts ST, Khanna AS, Barnabas RV, et al. Estimating the impact of universal antiretroviral therapy for HIV serodiscordant couples through home HIV testing: Insights from mathematical models.

20. Parker LA, Jobanputra K, Rusike L, et al. Feasibility and effectiveness of two community-based HIV testing models in rural Swaziland. Trop Med Int Health 2015;20(7):893-902. https://doi. org $/ 10.1111 /$ tmi. 12501

21. Joint United Nations Programme on HIV/AIDS (UNAIDS). A snapshot of men and HIV in South Africa. 2017. http://www.unaids.org/sites/default/files/snapshot-men-hiv-south-africa_en.pdf (accessed 7 November 2018).

22. Centers for Disease Control and Prevention. HIV Surveillance Report, 2016. Atlanta: CDC, 2017 